\section{Lab automation}

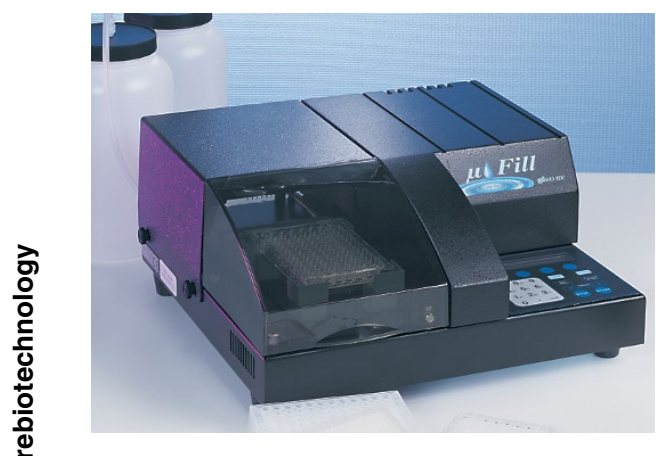

\section{Reagent dispenser}

Bio-Tek Instruments' $\mu$ Fill line of automated bulk reagent dispensers has added a 24-well microplate format. $\mu$ Fill incorporates a microprocessor-controlled syringe pump for optimal performance, and its compact footprint makes it ideally suited to fit inside laminar flow cabinets. Accurate, precise dispensing is guaranteed without the timeconsuming recalibration, cassette replacement and maintenance commonly associated with other dispensers. An autoclavable model is also available for those applications requiring a sterile fluid path.

http://www.biotek.com/

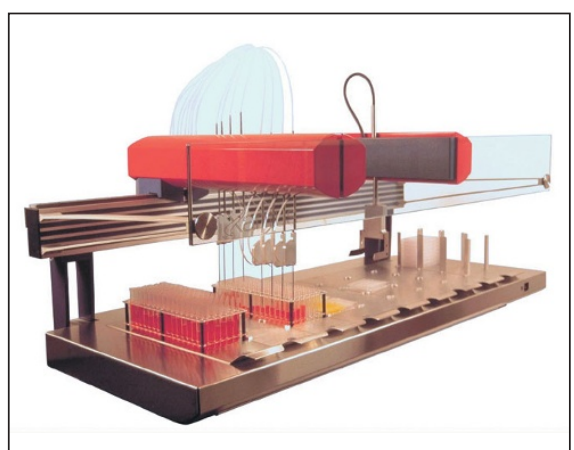

\section{Pipetting system}

The Sias Xantus combines flexible liquid handling with robotic manipulation. The modular design allows the hardware to be custom-configured and upgraded, and multi-level application software facilitates programming and optimization of even the most demanding applications. Xantus can have several arm modules, and each arm can carry up to eight pipetting tips or a robotic handler. With all functionality, including the pumps, housed in the arm, this entire module can be simply integrated into an OEM development.

http://www.sias.biz/

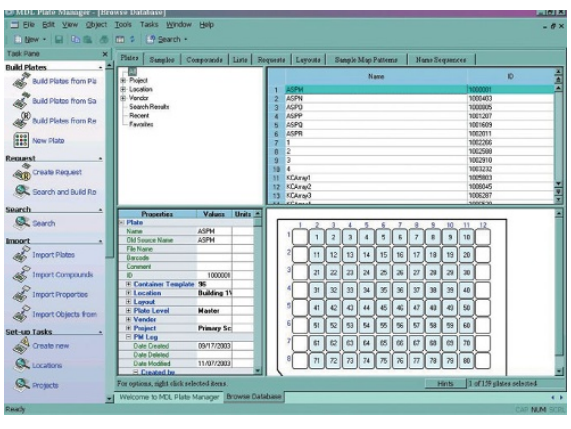

\section{Tracking samples}

MDL Information Systems has launched Plate Manager, a software solution for tracking, sorting and managing the vast amount of sample information generated in automated biopharmaceutical laboratories. Built on MDL's Isentris framework, Plate Manager improves pipeline productivity by resolving plate supply chain bottlenecks, ensuring sample integrity and data quality, and reducing cycle times and material inventories. Researchers can easily create new plates from existing plates or user-selected samples, reformat plate layouts, adjust concentrations or create dilution series, pool compounds into mixtures and cherry-pick hits. In addition, developers can customize the system to accommodate different workflows while also supporting straightforward integration with lab automation and third-party software.

http://www.mdl.com/

\section{Proteomics}

\section{High-content protein analysis}

BD Biosciences' FACSArray bioanalyzer for fast, sensitive, high-content analysis of proteins comprises an analyzer with a 96-well plate sampler, an optical detection unit, a computer workstation, acquisition and analysis software, and recommended applications. High sampling speed is achieved by combining novel plate sampler technology with digital electronics, generating acquisition rates up to 15,000 events per second. A 96-well plate can be run in less than 35 minutes, saving data for 1,000 events per well with each event reporting up to six parameters. A dual-laser optical detection system allows the combination of several bright fluorophores in one sample, enabling applications with a wide dynamic range. http://www.bdbiosciences.com/ 